\title{
Monodromy of an Inhomogeneous Picard-Fuchs Equation ${ }^{\star}$
}

\author{
Guillaume LAPORTE ${ }^{\dagger}$ and Johannes WALCHER ${ }^{\dagger \dagger}$ \\ $\dagger$ Department of Physics, McGill University, Montréal, Québec, Canada \\ E-mail: guillaume.laporte@mail.mcgill.ca \\ $\ddagger$ Department of Mathematics and Statistics, McGill University, Montréal, Québec, Canada \\ E-mail: johannes.walcher@mcgill.ca
}

Received June 08, 2012, in final form August 20, 2012; Published online August 22, 2012

http://dx.doi.org/10.3842/SIGMA.2012.056

\begin{abstract}
The global behaviour of the normal function associated with van Geemen's family of lines on the mirror quintic is studied. Based on the associated inhomogeneous Picard-Fuchs equation, the series expansions around large complex structure, conifold, and around the open string discriminant are obtained. The monodromies are explicitly calculated from this data and checked to be integral. The limiting value of the normal function at large complex structure is an irrational number expressible in terms of the di-logarithm.
\end{abstract}

Key words: algebraic cycles; mirror symmetry; quintic threefold

2010 Mathematics Subject Classification: 14C25; 14J33

\section{Introduction}

The early mathematical literature on mirror symmetry is replete with attempts to elucidate the enumerative predictions made by physics, [2] about rational curves on Calabi-Yau threefolds by utilizing the understanding of algebraic cycles on higher-dimensional varieties obtained from transcendental methods and deformation theory. The development of Gromov-Witten theory in the mid 1990's, culminating in a verification of the predictions, clarified the separation of the enumerative aspects of mirror symmetry (A-model) from the Hodge theoretic ones (B-model). Later, with the emergence of homological mirror symmetry (HMS) and the Strominger-YauZaslow (SYZ) picture, the classical questions had been all but driven out, and the enumerative aspects relegated to some challenging combinatorics of toric manifolds.

In recent years, it has become clear that algebraic cycles in fact have an eminent role to play in relating the established theory underlying classical mirror symmetry - Gromov-Witten theory and variation of Hodge structure, with the more elaborate versions such as HMS and SYZ. Indeed, if computing and comparing geometric invariants is the primary goal in elucidating the physics associated with Calabi-Yau manifolds, it is rather natural that algebraic cycles should be considered soon after the crudest topological data. They are obvious and well-studied algebraic invariants of the derived category, and have been proposed as mirrors to the (far less obvious, in fact not yet defined) on-shell invariants of the Fukaya category.

On the other hand, when viewed against the backdrop of the intricacies of the string duality web, and the fact that some of the deepest conjectures in algebraic geometry concern algebraic cycles, the speculation that the entire enumerative geometry of Calabi-Yau manifolds (GromovWitten, BPS, and otherwise) might be encoded in the (higher) Chow groups of the mirror manifold seems rather stably grounded. The earliest reference to such ideas that we are aware of is made in [4].

\footnotetext{
*This paper is a contribution to the Special Issue "Mirror Symmetry and Related Topics". The full collection is available at http://www.emis.de/journals/SIGMA/mirror_symmetry.html
} 
A most intriguing consequence of this basic assumption arises via the fundamentally arithmetic nature of algebraic cycles. Even if (as would be natural for a physicist, interested in the real world or not), one is expecting to deal primarily with complex numbers, the field of definition of the algebraic cycle relative to that of the underlying variety invariably enters the discussion in all but the very simplest situations. Whether this is a mere curiosity in a rather special setup, or the hint of a more significant connection between the two subjects, it is clear that some adjustement of the physical picture will have to take place. And quite similarly, symplectic geometers should need to contemplate arithmetic considerations playing an important role in a detailed study of certain Fukaya categories and their deformations.

The exploration of these questions was taken up in the recent paper [8], using explicitly constructed algebraic cycles on the mirror quintic threefold. Without identifying the precise A-model setup, it was found that the would-be enumerative invariants are in general algebraic numbers satisfying a certain integrality condition. Actually, the (irrational) algebraicity of the relevant expansion coefficients is an inevitable consequence of the fact that the irreducible components of the algebraic cycle are defined over an extension of the field of definition of the underlying manifold (in this case, the field $\mathbb{Q}$ of rational numbers). The meaning of the integrality and the A-model or spacetime physics explanation for the irrationality of the enumerative invariants, remains to be found.

A different type of arithmeticity of the same Hodge theoretic invariants of algebraic cycles has been recently explained by Griffiths, Green and Kerr, see [6]. In certain degenerate limits, including those of relevance for mirror symmetry, standard Abel-Jacobi mappings such as those underlying the calculations of the D-brane superpotential carried out in [8] turn out to be given by higher Abel-Jacobi mappings on certain higher Chow groups. In turn, these degenerate Abel-Jacobi mappings are regulators on certain K-groups of the singular member of the family, and have an arithmetic significance. This was illustrated in [6] in various examples, including one closely related to the one we study here.

In this paper, we will report on some further calculations around one of the algebraic cycles on the mirror quintic studied in [8]. Starting from the inhomogeneous Picard-Fuchs equation satisfied by the associated normal function, we will compute the expansion of that normal function around the various interesting points in the complex structure moduli space. By comparing (numerically) these various expansions, we will be able to determine the complete analytic continuation of the normal function around the entire moduli space. In particular, this will provide the transformation under mondromy around the singular loci. The monodromies will be integral, as they should be on general grounds. This can be viewed as a consistency check on the normalization of the normal function found in [8]. (This check is satisfying, but honestly redundant, as the normalization is completely determined by the methods used in [8].)

Our calculations will also determine a constant of integration that was not calculated in [8]. This interesting constant is the only one not constrained to be rational by monodromy considerations. It is the limiting value of the normal function, and precisely the one number which the invariants of [6] reduce to for co-dimension 2 cycles on Calabi-Yau threefolds. Confirming general expectations, and results of [6], we will find that this constant is, up to a rational multiple, given by the special value of an L-function of the algebraic number field over which the underlying cycle is defined.

\section{Equations}

We start from the vanishing locus $\{W=0\}$ of the familiar family of polynomials

$$
W=x_{1}^{5}+x_{2}^{5}+x_{3}^{5}+x_{4}^{5}+x_{5}^{5}-5 \psi x_{1} x_{2} x_{3} x_{4} x_{5}
$$




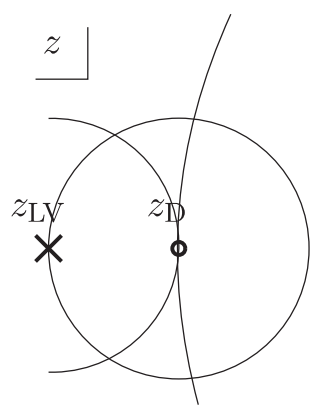

$\infty$

$\times$

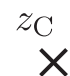

Figure 1. Moduli space of the mirror quintic with van Geemen family. The arcs indicate the circles of convergence of the series expansions about the various singular points.

in $\mathbb{P}^{4} \ni\left[x_{1}: x_{2}: x_{3}: x_{4}: x_{5}\right]$. The mirror quintic family is the resolution of the quotient

$$
Y=\{W=0\} /\left(\mu_{5}\right)^{3},
$$

where $\left(\mu_{5}\right)^{3} \cong(\mathbb{Z} / 5 \mathbb{Z})^{3}$ is the Greene-Plesser group of phase symmetries leaving $W$ invariant. As $\psi$ varies, it parameterizes local deformations of the complex structure of the mirror quintic. Multiplication of $\psi$ by a fifth root of unity can be undone by a change of coordinates on $\mathbb{P}^{4}$, and a good global coordinate on the moduli space is the familiar

$$
z=(5 \psi)^{-5} \text {. }
$$

Our moduli space has three distinguished points: the large volume point, corresponding to $z=$ $z_{\mathrm{LV}}=0$, the conifold point at $z=z_{\mathrm{C}}=5^{-5}$, and the orbifold (or Gepner) point, $z^{-1}=z_{\mathrm{G}}{ }^{-1}=0$, see Fig. 1 for a sketch.

The convenient choice of holomorphic three-form on $Y$ comes from

$$
\Omega=\left(\frac{5}{2 \pi i}\right)^{3} \operatorname{Res}_{W=0} \frac{\sum_{i=1}^{5}(-1)^{i} x_{i} d x_{1} \wedge \cdots \wedge \widehat{d x}_{i} \wedge \cdots d x_{5}}{W} .
$$

Then, for any $[\Gamma] \in H_{3}(Y, \mathbb{Z}) \cong \mathbb{Z}^{4}$, the corresponding period

$$
\varpi(z)=\int_{\Gamma} \Omega
$$

satisfies as a function of $z$ the differential equation

$$
\mathcal{L} \varpi(z)=0
$$

where $\mathcal{L}$ is the Picard-Fuchs differential operator

$$
\mathcal{L}=\theta^{4}-5 z(5 \theta+1)(5 \theta+2)(5 \theta+3)(5 \theta+4), \quad \theta \equiv \frac{z d}{d z} .
$$

Now let $\omega$ be a non-trivial third root of unity, i.e., a solution of the quadratic equation

$$
1+\omega+\omega^{2}=0
$$

Then, as one may check, the holomorphic line in $\mathbb{P}^{4}$ given by the equations

$$
x_{1}+\omega x_{2}+\omega^{2} x_{3}=0, \quad a\left(x_{1}+x_{2}+x_{3}\right)=3 x_{4}, \quad b\left(x_{1}+x_{2}+x_{3}\right)=3 x_{5}
$$


is contained in the hypersurface $\{W=0\}$, if and only if, for any $\psi, a$ and $b$ form a solution to the equations

$$
a b \psi=6, \quad a^{5}+b^{5}=27 .
$$

The lines given by equations (4) subject to equations (5), and their images under the group of discrete symmetries of $W$ are known as van Geemen lines and have been fruitfully studied over the years, see in particular $[1,7]$. One of the results that is relevant for us is that a van Geemen line at fixed $\psi$ belongs to one of two families of lines that can be distinguished by exchanging $a$ and $b$ or, equivalently, replacing $\omega$ with $\omega^{2}$ in equation (3).

Passing the orbit of a van Geemen line under the Greene-Plesser group $\left(\mu_{5}\right)^{3}$ through the resolution of singularities induces a family of algebraic cycles on the mirror quintic $Y$ that we shall denote by $C_{\omega}$. The truncated normal function associated with this family of cycles is the integral

$$
\tau(z)=\int_{C_{\omega}}^{C_{\omega^{2}}} \Omega
$$

of the holomorphic three-form against a three-chain bounding $C_{\omega^{2}}-C_{\omega}$. This integral was shown in [8] to satisfy the inhomogeneous Picard-Fuchs equation

$$
\mathcal{L} \tau(z)=\frac{1+2 \omega}{(2 \pi i)^{2}} \cdot \frac{32}{45} \cdot \frac{\frac{63}{\psi^{5}}+\frac{1824}{\psi^{10}}-\frac{512}{\psi^{15}}}{\left(1-\frac{128}{3 \psi^{5}}\right)^{5 / 2}} .
$$

The characteristic denominator originates from the discriminant of the equations (5). This discriminant consists of the Gepner point, $\psi=0$, as well as the locus $z=z_{\mathrm{D}}=3 \cdot 2^{-7} \cdot 5^{-5}$. We will sometimes refer to the latter as the "open string discriminant", keeping in mind that the Gepner point is the other branch point of the quadratic extension of the moduli space by the inhomogeneity. Note in particular that while at fixed $\psi$ or $z$, we have two distinguished groups of van Geemen lines, monodromy around $z_{\mathrm{D}}$ exchanges the two branches that we labelled $C_{\omega}$ and $C_{\omega^{2}}$. This will be crucial for what follows.

We now describe the problem that we will solve in this work. The differential equation (1) admits four solutions that are linearly independent over $\mathbb{C}$. Solutions corresponding to an integral basis of $H_{3}(Y, \mathbb{Z})$ are however well-known. Such a basis was first obtained in [2] by calculating the transformation properties of the solutions under monodromy around the moduli space, and has played an important role in several subsequent developments. Later, the integral variations of Hodge structure underlying these calculations have been identified and fully classified in this case, see [5], which fact provides a firm basis for our present discussion.

A similar issue afflicts the inhomogeneous equation (7). While $\tau(z)$ is initially defined in (6) by the choice of chain up to an integral three-cycle, the differential equation determines $\tau(z)$ only up to a complex linear combination of periods. By the same token, encircling one of the singularities in the moduli space must return the chain up to an integral cycle so $\tau(z)$ must transform by an integral period. Note that the availability of the above-mentioned integral lift of monodromy is crucial for asking a sensible question here.

We will fix the choice of chain equivalent to imposing specific boundary conditions at the open string discriminant $z_{\mathrm{D}}$. As $z$ approaches $z_{\mathrm{D}}$, pairs of solutions of (5) will approach each other. In $Y$, this means that the associated van Geemen lines will coincide, and we may choose to define $\tau(z)$ using the corresponding vanishing three-chain. Analytically, this means that $\tau(z)$ as well as its first derivative $\tau^{\prime}(z)$ vanish at $z=z_{\mathrm{D}}$. (The first derivative with respect to $z$ gives the integral of the first order deformation of the holomorphic 3-form, and must vanish together with the integral of $\Omega$ itself.) To see that this condition is compatible with the behaviour of the 
inhomogeneity in (7), we note that in a local coordinate $y=z-z_{\mathrm{D}}$, the Picard-Fuchs equation will take the leading order form

$$
\mathcal{L} \tau \sim \frac{d^{4}}{d y^{4}} \tau \propto y^{-5 / 2}
$$

so the solution $\tau \sim y^{3 / 2}$ as well as $\tau^{\prime} \sim y^{1 / 2}$ will vanish as $y \rightarrow 0$. In fact, since as noted above, monodromy around $y=0$ exchanges $C_{\omega}$ with $C_{\omega^{2}}$, our $\tau$ is affected precisely by a change of sign. Thus, the condition that $\tau / y^{3 / 2}$ be analytic at $y=0$ completely fixes the solution of the inhomogeneous equation (7).

Our main results are $(i)$ the verification that under this boundary condition at $z_{\mathrm{D}}$, the monodromies of $\tau$ around the other singular points are indeed integral, and ( $i i)$ the calculation of the leading asymptotic behaviour at the large volume point. General theory, see [6], restricts the limiting values of normal functions under degenerations such as that at $z_{\mathrm{LV}}$, and we will find that our results are compatible with these restrictions.

Calculations similar to the one described here were carried out for a different class of algebraic cycles on the mirror quintic in [9].

\section{Expansions}

In this section, we collect the expansions of the solutions of the homogeneous and inhomogeneous Picard-Fuchs equation around the various distinguished points in moduli space. We begin at large volume.

Around $z=z_{\mathrm{LV}}$, a basis of solutions of equation (1) can be generated using Frobenius method from the hypergeometric series

$$
\varpi(z ; H)=\sum_{n=0}^{\infty} \frac{\Gamma(5(n+H)+1)}{\Gamma(n+H+1)^{5}} z^{n+H} .
$$

Indeed, since $\mathcal{L} \varpi(z ; H) \propto H^{4}$, and $\left[\partial_{H}, \mathcal{L}\right]=0$, a basis of solutions is given by

$$
\varphi_{k}(z)=\left.\frac{1}{(2 \pi i)^{k}}\left(\partial_{H}\right)^{k}\right|_{H=0} \varpi(z ; H), \quad k=0,1,2,3,
$$

with leading behaviour

$$
\begin{array}{ll}
\varphi_{0}(z)=f_{0}, & f_{0}=1+120 z+113400 z^{2}+\cdots, \\
(2 \pi i) \varphi_{1}(z)=f_{0} \log z+f_{1}, & f_{1}=770 z+810225 z^{2}+\cdots, \\
(2 \pi i)^{2} \varphi_{2}(z)=f_{0} \log ^{2} z+2 f_{1} \log z+f_{2}, & f_{2}=\frac{2}{5} \cdot 2875 z+\frac{4208175}{2} z^{2}+\cdots, \\
(2 \pi i)^{3} \varphi_{3}(z)=f_{0} \log ^{3} z+3 f_{1} \log ^{2} z+3 f_{2} \log z+f_{3}, & f_{3}=-\frac{12}{5} \cdot 2875 z-\frac{9895125}{2} z^{2}+\cdots
\end{array}
$$

This basis is close to being integral, but there are some important rational as well as irrational modifications. By results of [2], a (projectively) integral basis is given by the period vector $\Pi=\left(\varpi_{0}, \varpi_{1}, \varpi_{2}, \varpi_{3}\right)^{T}$ with

$$
\begin{aligned}
& \varpi_{0}(z)=\varphi_{0}(z), \quad \varpi_{1}(z)=\varphi_{1}(z), \quad \varpi_{2}(z)=\frac{5}{2} \varphi_{2}(z)-\frac{5}{2} \varphi_{1}(z)-\frac{25}{12} \varphi_{0}(z), \\
& \varpi_{3}(z)=-\frac{5}{6} \varphi_{3}(z)-\frac{25}{12} \varphi_{1}(z)+200 \frac{\zeta(3)}{(2 \pi i)^{3}} \varphi_{0}(z) .
\end{aligned}
$$


Under $z \rightarrow e^{2 \pi i} z$, we have $\varphi_{k} \rightarrow \sum_{j=0}^{k}\left(\begin{array}{c}k \\ j\end{array}\right) \varphi_{j}$ and thereby, large volume monodromy is represented on the period vector

$$
\Pi \rightarrow M_{\mathrm{LV}} \Pi
$$

by the matrix

$$
M_{\mathrm{LV}}=\left(\begin{array}{cccc}
1 & 0 & 0 & 0 \\
1 & 1 & 0 & 0 \\
0 & 5 & 1 & 0 \\
-5 & 5 & 1 & 1
\end{array}\right)
$$

We draw attention to the one irrational constant in equation (8),

$$
200 \frac{\zeta(3)}{(2 \pi i)^{3}} \approx i \cdot 0.9692044901 \ldots,
$$

which decouples from monodromy considerations at large volume, but can be determined by a close examination of the conifold locus (see [2]).

In the local variable $w=1-5^{5} z$, which vanishes at $z=z_{\mathrm{C}}$ we may work out a basis of solutions of equation (1) to be given by

$$
\begin{aligned}
& \psi_{0}=1+\frac{2}{625} w^{3}+\frac{97}{18750} w^{4}+\cdots, \\
& \psi_{1}=\frac{\sqrt{5}}{2 \pi i} \cdot\left(-w-\frac{7}{10} w^{2}-\frac{41}{75} w^{3}-\frac{1133}{2500} w^{4}-\cdots\right), \\
& \psi_{2}=\frac{1}{2 \pi i} \psi_{1} \log w+\frac{\sqrt{5}}{\pi^{2}} \cdot\left(-\frac{23}{1440} w^{3}-\frac{6397}{240000} w^{4}-\cdots\right), \\
& \psi_{3}=w^{2}+\frac{37}{30} w^{3}+\frac{2309}{1800} w^{4}-\cdots,
\end{aligned}
$$

and assemble it into the conifold period vector $\Psi=\left(\psi_{0}, \psi_{1}, \psi_{2}, \psi_{3}\right)$. We emphasize that this is not an integral basis of periods. In fact, it is not known analytically how the integral basis (8) is related to the basis (10). It is known however, and this explains the choice of integration constants, that $\varpi_{3}=\psi_{1}$, and that $\varpi_{0}-\psi_{2}, \varpi_{1}, \varpi_{2}$ are linear combinations of $\psi_{0}, \psi_{1}, \psi_{3}$. In other words, the conifold monodromy $w \rightarrow e^{2 \pi i} w$ is represented on the large volume period vector as

$$
M_{\mathrm{C}}=\left(\begin{array}{cccc}
1 & 0 & 0 & 1 \\
0 & 1 & 0 & 0 \\
0 & 0 & 1 & 0 \\
0 & 0 & 0 & 1
\end{array}\right)
$$

These results were originally obtained in [2] by exploiting the analytic continuation to the Gepner point, which can be done in closed form. Since to reach the open string discriminant, we will have to resort to numerical continuation anyway, we will skip this part of the story. We only record that on the periods, monodromy around the Gepner point is equivalent to the composition of large volume and conifold monodromy. It is therefore represented by the order 5 matrix

$$
M_{\mathrm{G}}=M_{\mathrm{LV}} \cdot M_{\mathrm{C}}=\left(\begin{array}{cccc}
1 & 0 & 0 & 1 \\
1 & 1 & 0 & 1 \\
0 & 5 & 1 & 0 \\
-5 & -5 & -1 & -4
\end{array}\right), \quad\left(M_{\mathrm{G}}\right)^{5}=\mathbf{1} .
$$

Turning then to the inhomogeneous equation (7), we find the power series expansion of a solution around large volume

$$
\tau_{\mathrm{LV}}(z)=\frac{\sqrt{-3}}{\pi^{2}} \cdot\left(140000 z+\frac{11521900000}{3} z^{2}+\frac{5187112292000000}{27} z^{3}+\cdots\right)
$$


and conifold

$$
\tau_{\mathrm{C}}(w)=\frac{\sqrt{5}}{\pi^{2}} \cdot\left(\frac{88}{15625} w^{3}+\frac{4282}{390625} w^{4}+\cdots\right) .
$$

Finally, around the open string discriminant, $z=z_{\mathrm{D}}$ we use the variable $y=1-2^{7} \cdot 5^{5} \cdot 3^{-1} z$. As we have explained above, we fix boundary conditions such that $\tau / y^{3 / 2}$ is analytic as $y \rightarrow 0$. This solution has the power series expansion

$$
\tau(y)=\frac{\sqrt{-3}}{\pi^{2}} \cdot\left(\frac{16}{5} y^{3 / 2}+\frac{8352}{3125} y^{5 / 2}+\frac{6156432}{2734375} y^{7 / 2}+\cdots\right) .
$$

\section{Continuations}

The power series (11), (12), and (13) all represent solutions of the inhomogeneous Picard-Fuchs equation (7). These solutions must be equal modulo a solution of the homogeneous equation, i.e., a period. As we have explained, the solution of our interest is determined by the boundary condition at the open string discriminant, i.e., $\tau \sim y^{3 / 2}$, and we wish to relate it to the other two expansions. (The continuation to the Gepner point can then be inferred from this data.)

Our first task then is to express $\tau$ in terms of $\tau_{\mathrm{LV}}$ and $\Pi=\left(\varpi_{0}, \varpi_{1}, \varpi_{2}, \varpi_{3}\right)$. For this aim, we note that the power series (11) and (13) have a radius of convergence equal to $\left|z_{\mathrm{D}}\right|$, the latter because of the singularity of the differential operator (2), and the former because of the apparent singularity in the inhomogeneity (7) (cf. Fig. 1). Both expansions, as well as (8), converge well at the midpoint, $z=z_{\mathrm{D}} / 2$, which is therefore a convenient point to compare. By evaluating numerically all those functions and their derivatives, we find that

$$
\tau=\tau_{\mathrm{LV}}+v_{\mathrm{LV}} \cdot \Pi
$$

where

$$
v_{\mathrm{LV}}=(a,-10,0,2),
$$

with

$$
a \approx i \cdot 13.36856103560663627 \ldots
$$

It is not quite that straightforward to compare $\tau_{\mathrm{C}}$ with $\tau$. While equation (12) converges up to the open string discriminant, the expansion (13) still has radius of convergence $\left|z_{\mathrm{D}}\right|$, which is much less than the distance $\left|z_{\mathrm{C}}-z_{\mathrm{D}}\right|$. A comparison would of course be possible in the intersection of the two disks, but (12) does not converge well in this region. Instead, we resort to solving the differential equation numerically, with initial conditions around $z_{\mathrm{D}}$ given by $\tau$, and compare with $\tau_{\mathrm{C}}$ close to $z_{\mathrm{C}}$. We find

$$
\tau=\tau_{\mathrm{C}}+\tilde{v} \cdot \Psi
$$

where $\tilde{v}=(3.3655477 \ldots, i \cdot 2.9120714 \ldots, 8,0.18666620 \ldots)$ is a certain vector with one integral and three apparently irrational entries. Since $\Psi$ is not fully integral, it appears more significant, and more convenient to compute monodromies, to give the relation to the integral large volume basis, namely,

$$
v_{\mathrm{C}}=\tilde{v} \Psi \cdot \Pi^{-1}=\left(8, c_{1}, c_{2}, c_{3}\right),
$$

where $c_{1} \approx 9.197317 \cdots-i \cdot 13.598567 \ldots, c_{2} \approx 3.6789269 \ldots, c_{3} \approx-i \cdot 2.3915634 \ldots$ 
With all this data in hand, it is now easy to write down the transformation properties of our distinguished solution $\tau$ under monodromy about the various singularities. Using (14) and the fact that $v_{\mathrm{LV}}$ is single valued around large volume, we obtain

$$
\tau \rightarrow \tau+m_{\mathrm{LV}} \cdot \Pi
$$

with

$$
m_{\mathrm{LV}}=v_{\mathrm{LV}} \cdot M_{\mathrm{LV}}-v_{\mathrm{LV}}=(-20,-10,2,0) .
$$

About the conifold, we exploit invariance of $\tau_{\mathrm{C}}$ to find that

$$
\tau \rightarrow \tau+m_{\mathrm{C}} \cdot \Pi
$$

with

$$
m_{\mathrm{C}}=v_{\mathrm{C}} \cdot M_{\mathrm{C}}-v_{\mathrm{C}}=(0,0,0,8) .
$$

And, of course, monodromy around the open string discriminant is, by construction

$$
\tau \rightarrow-\tau \text {. }
$$

Putting this together, we may infer also the monodromy around the Gepner point

$$
\tau \rightarrow-\tau+m_{\mathrm{G}} \cdot \Pi
$$

with

$$
m_{\mathrm{G}}=m_{\mathrm{C}}+m_{\mathrm{LV}} \cdot M_{\mathrm{C}}=(-20,-10,-2,-12) .
$$

One may also check that the order of the Gepner monodromy has been extended from 5 to 10 , rather as in [9].

\section{Discussion}

The basic result of the previous section is the integrality of the vectors $m_{\mathrm{LV}}, m_{\mathrm{C}}, m_{\mathrm{G}}$. Under monodromy around the moduli space, the truncated normal function comes back to itself (or minus itself, if one encloses the open string discriminant or Gepner point, which are the branch points of the inhomogeneity equation (7)), up to an integral period. It remains to discuss the significance of the one irrational entrie in $v_{\mathrm{LV}}$, given in equation (16).

The general theory of limiting values of normal functions under degeneration of the underlying variation of Hodge structure has been recently explained by Griffiths, Green and Kerr [6]. This work provides, first of all, the identification of the Abelian group "filling in" for the intermediate Jacobian at the singular fiber of a degenerating Hodge structure. These "Néron models" then allow the discussion of the limiting values of normal functions. And for a normal function coming from geometry (i.e., an algebraic cycle), this limiting value is interpreted as the Abel-Jacobi map on a certain motivic cohomology of the singular fiber. An important point is that the theory of [6] deals with the phenomena in the strict degeneration limit, whereas we have been concerned here with the analytic expansion of the normal function around such a limit.

In somewhat simplified language, and specialized to the case of our interest (co-dimension 2 cycles on a one-parameter family of Calabi-Yau threefolds, in a maximal unipotent degeneration) the main result of [6] states that, in the limit $z \rightarrow 0$, any normal function arising from an algebraic cycle must asymptote to the period of the one monodromy-invariant three-cycle. Remembering 
that this is just the fundamental period $\varpi_{0}(z)$, the result we found in equation (15) is in precise agreement with that condition. (All integral periods would disappear under the map to the intermediate Jacobian.) Intuitively, one may understand the limiting condition from the requirement that the monodromy be integral. In this respect, the coefficient $a$ is very similar to the constant of equation (9) appearing in the expansion of the ordinary periods. It should also be pointed out that the condition holds as stated under the assumption that the algebraic cycle itself is invariant under the large volume monodromy. Otherwise, one might pass to an appropriate cover of the moduli space, with results similar to those in [9].

It is also explained in [6] that the limiting value of the normal function (i.e., the coefficient of the fundamental period) has an arithmetic significance. Namely, our $a$ is the image of the limiting cycle under a certain Bloch regulator map. As such, it should be expressible in terms of the Bloch-Wigner function

$$
D(z)=\operatorname{Im}\left(\operatorname{Li}_{2}(z)\right)+\arg (1-z) \log |z| .
$$

Indeed, guided by the example of [6], we find (numeratically), that

$$
a=\frac{195}{\pi^{2}} D\left(e^{2 \pi i / 3}\right) .
$$

An alternative expression follows from the relation between special values of the Bloch-Wigner function and special values of L-functions of algebraic number fields, see [10]. In the case at hand, we recall that the definition of our van Geemen lines involved from the very beginning that number field $\mathbb{Q}(\sqrt{-3})$. The corresponding Dirichlet L-function is

$$
L(s)=\sum_{k=1}^{\infty}\left(\frac{k}{3}\right) \frac{1}{k^{s}}
$$

and using formulas of [10], we obtain

$$
a=\frac{195 \sqrt{-3}}{2 \pi^{2}} L(2) \text {. }
$$

The main reason for giving this alternate formula is that it allows making contact with the results of [8] concerning the arithmetic properties of the actual expansion of the normal function around $z=0$ (as opposed to just its limiting value). Indeed, it was observed that to exhibit the underlying integrality of that expansion under the mirror map, one has to twist the standard Ooguri-Vafa di-logarithm multi-cover formula precisely as in equation (18). This was dubbed the D-logarithm,

$$
\operatorname{Li}_{2}^{(\chi)}(z)=\sum_{k=1}^{\infty}\left(\frac{k}{3}\right) \frac{z^{k}}{k^{2}}
$$

with the obvious coincidence of special values $\mathrm{Li}_{2}^{(\chi)}(1)=L(2)$. We do not know whether these relations generalize.

To close, we point out two obvious questions that deserve immediate attention. First of all, one should verify the formula (17) or (19) from direct geometric considerations, closely related to the example of [6]. Secondly, one should aim to understand the origin of these special values from the A-model or space-time physics perspective. There are two rather plausible explanations: From the point of view of a Lagrangian D-brane that is mirror to the algebraic cycle we have been considering, the constant $a$ should be related to a certain perturbative invariant in the ChernSimons theory living on that D-brane. These invariants are known to take arithmetric values, 
see e.g., [3]. Lastly, from the point of view of the topological sigma-model, $a$ should be related to a perturbative $\alpha^{\prime}$ correction to the bosonic potential of the Kähler moduli fields induced by the presence of the D-brane. This would be similar to the way that the constant $\chi(X) \zeta(3) / \pi^{3}$ in (9) arises from a four-loop correction to the sigma-model metric on the background Calabi-Yau $X$, see [2]. To the best of our knowledge, the corresponding open string calculation has not yet been done.

\section{Acknowledgements}

We would like to thank Matt Kerr for asking the question addressed in this work, and Josh Lapan for stimulating discussions. J.W. wishes to thank the Simons Center for Geometry and Physics, where this paper was written up. This work was supported in part by the Canada Research Chair program and an NSERC discovery grant.

\section{References}

[1] Albano A., Katz S., Lines on the Fermat quintic threefold and the infinitesimal generalized Hodge conjecture, Trans. Amer. Math. Soc. 324 (1991), 353-368.

[2] Candelas P., de la Ossa X.C., Green P.S., Parkes L., A pair of Calabi-Yau manifolds as an exactly soluble superconformal theory, Nuclear Phys. B 359 (1991), 21-74.

[3] Dimofte T., Gukov S., Lenells J., Zagier D., Exact results for perturbative Chern-Simons theory with complex gauge group, Commun. Number Theory Phys. 3 (2009), 363-443, arXiv:0903.2472.

[4] Donagi R., Markman E., Cubics, integrable systems, and Calabi-Yau threefolds, in Proceedings of the Hirzebruch 65 Conference on Algebraic Geometry (Ramat Gan, 1993), Israel Math. Conf. Proc., Vol. 9, Bar-Ilan Univ., Ramat Gan, 1996, 199-221, alg-geom/9408004.

[5] Doran C.F., Morgan J.W., Mirror symmetry and integral variations of Hodge structure underlying oneparameter families of Calabi-Yau threefolds, in Mirror Symmetry. V, AMS/IP Stud. Adv. Math., Vol. 38, Amer. Math. Soc., Providence, RI, 2006, 517-537.

[6] Green M., Griffiths P., Kerr M., Néron models and limits of Abel-Jacobi mappings, Compos. Math. 146 (2010), 288-366.

[7] Mustaţă A., Degree 1 curves in the Dwork pencil and the mirror quintic, Math. Ann., to appear, math.AG/0311252.

[8] Walcher J., On the arithmetics of D-brane superpotentials, arXiv:1201.6427.

[9] Walcher J., Opening mirror symmetry on the quintic, Comm. Math. Phys. 276 (2007), 671-689, hep-th/0605162.

[10] Zagier D., The dilogarithm function, in Frontiers in Number Theory, Physics, and Geometry. II, Springer, Berlin, 2007, 3-65. 